\title{
Yersinia enterocolitica Infection of a Prosthetic Knee Joint. Case Report and Review of the Literature on Deep Sited Infections Caused by Y. enterocolitica
}

\author{
Päivi Jalava-Karvinen ${ }^{1,2}$, Jarmo Oksi ${ }^{1,2}$, Kaisu Rantakokko-Jalava ${ }^{3}$, Petri Virolainen ${ }^{2,4}$, \\ Pirkko Kotilainen ${ }^{1,2}$ \\ ${ }^{1}$ Department of Infectious Diseases, Division of Medicine, Turku University Hospital, Turku, Finland; ${ }^{2}$ Faculty of Medicine, Turku \\ University, Turku, Finland; ${ }^{3}$ Clinical Microbiology Laboratory, Turku, Finland; ${ }^{4}$ Department of Orthopedics and Traumatology, \\ Turku University Hospital, Turku, Finland. \\ Email: paijal@utu.fi
}

Received February $15^{\text {th }}, 2013$; revised March $28^{\text {th }}$, 2013; accepted April $28^{\text {th }}, 2013$

Copyright (C) 2013 Päivi Jalava-Karvinen et al. This is an open access article distributed under the Creative Commons Attribution License, which permits unrestricted use, distribution, and reproduction in any medium, provided the original work is properly cited.

\begin{abstract}
Prosthetic joint infection is a rare manifestation of Yersinia enterocolitica. We report a case of a patient presenting with fever and a purulent infection in his prosthetic knee joint caused by Y. enterocolitica. He had been operated in 1990 for arthrosis of the right knee. Re-operation was performed in 2007 for loosening of the prosthesis. Seven months later, following progressively increasing knee pain, he became acutely febrile and a purulent knee joint infection was diagnosed. $Y$. enterocolitica was isolated from the joint fluid. Serum antibodies against $Y$. enterocolitica were also positive. He was treated with debridement, replacement of the liner component of the prosthesis and a long course of intravenous antimicrobial therapy. The infection was thought to be in a chronic suppressive state. The final outcome after all therapy was good.
\end{abstract}

Keywords: Debridement Operation; Prosthetic Knee Joint; Purulent Infection; Yersinia enterocolitica

\section{Introduction}

Yersinia enterocolitica is a gram-negative rod-shaped bacterium that typically causes gastrointestinal infections, and occasionally leads to reactive inflammatory arthritis of one or a few joints [1,2]. Reactive arthritis follows the primary infection after a period of one to three weeks. Typically, the causative agent cannot be eradicated from the joint fluid at the time of arthritis. Y. enterocolitica is a rare cause of deep purulent infections. Only a few cases of patients with purulent prosthetic infections caused by $Y$. enterocolitica have been reported in the English literature. There are only two previous reports on prosthetic knee joint infection caused by $Y$. enterocolitica $[3,4]$. We describe a case of a patient with a purulent infection in his prosthetic knee joint caused by $Y$. enterocolitica.

\section{Case Report}

A 90-year-old Finnish man with hypertension had a history of coronary artery by-pass operation performed thirteen years earlier. He was on warfarin treatment for pre- vention of thromboembolic complications of atrial fibrillation. A right knee joint replacement with a MillerGalante prosthesis was performed in 1990 because of degenerative changes in the joint. His health was good despite his advanced age, and he still had and actively used his driver's licence. He had long been satisfied with his prosthetic knee joint but gradually, more than fifteen years later, the joint had become painful and a metallic sound could be heard during walking. In February 2007 he was re-operated and severe metallosis and osteolysis were found in the prosthetic joint. Re-arthroplasty was performed with Duracon TS components and the primary outcome as well as recovery was normal. He was well two months after the operation, with no signs of infection reported at the time of first post-operative control examination.

In September 2007 he developed a sudden fever up to $39^{\circ} \mathrm{C}$ and the right prosthetic knee became inflamed with swelling, redness and pain. During the previous three months, the knee had become progressively painful, finally causing him difficulties in weight bearing. He had 
had no preceding gastrointestinal symptoms. The joint was punctured by a primary health care physician and bloody pus-containing joint fluid was aspirated. Ceftriaxone was administered intravenously and the patient was sent to our tertiary care hospital.

On admission, the patient had a fever of $38.6^{\circ} \mathrm{C}$ and the affected knee was painful, swollen, reddish, and warm. His hemodynamic state was stable with blood pressure 131/67 $\mathrm{mmHg}$ and heart rate 93/min. C-reactive protein (CRP) was $138 \mathrm{mg} / \mathrm{l}$, hemoglobin $148 \mathrm{~g} / \mathrm{l}$, leucocytes $11.7 \times 10^{9} / l$, and thrombocytes $93 \times 10^{9} /$ l. Erythrocyte sedimentation rate (ESR) was $52 \mathrm{~mm} / \mathrm{h}$. X-ray imaging of the right knee showed the prosthesis located in the correct position. No signs of loosening of the prosthesis were seen. Next day, a debridement operation was performed with lavation and exchange of the polyethylene liner component of the endoprosthesis. Because of the advanced age of the patient, total re-arthroplasty was not performed. In the sample aspirated by the primary health care physician, $Y$. enterocolitica was isolated. The isolate was identified by Vitek 2 GN identification card with excellent confidence level (99\%) and API $20 \mathrm{E}$ with profile 0014523 (both from bioMèrieux, Marcy L'Etoile, France). The identification and the serotype were confirmed by the national reference laboratory. The organism was sensitive to amikacin, amoxicillin + clavulanate, piperacillin + tazobactam, tobramycin, co-trimoxazole, ciprofloxacin, ceftazidime, ceftriaxone, cefuroxime, and imipenem, and resistant to ampicillin and cephalotin. Serum antibodies against $Y$. enterocolitica serotype 3 (Y.ent3AbM+++, AbA+++, IgG++) reflected a recent $Y$. enterocolitica serotype 3 infection.

The treatment had been started with ceftriaxone $2 \mathrm{~g}$ once daily but after seven days treatment was switched to piperacillin + tazobactam $4 \mathrm{~g}$ t.i.d. on the basis of the clinical picture. Two days later, according to sensitivity testing and a literature survey, ceftriaxone was reinstituted along with oral ciprofloxacin $500 \mathrm{mg}$ b.i.d.

Because no clinical improvement was seen after four weeks, ceftriaxone was changed to meropenem $1 \mathrm{~g}$ t.i.d., with the oral ciprofloxacin $500 \mathrm{mg}$ b.i.d. continued. However, the CRP level had decreased and was then within the normal range $(9 \mathrm{mg} / \mathrm{l}$, normal $<10 \mathrm{mg} / \mathrm{l})$.The $\mathrm{X}$-ray images of the joint showed radiolucency between the bone and cement around the prosthesis. Bloody puslike joint fluid was aseptically aspirated three times (30 $60 \mathrm{ml}$ each time) from the prosthetic joint on the advice of an orthopedist. The knee was warm, swollen and painful but the redness had disappeared. After treatment with intravenous meropenem in combination with ciprofloxacin $500 \mathrm{mg}$ b.i.d. for six weeks, the patient was discharged with oral ciprofloxacin $750 \mathrm{mg}$ b.i.d. The CRP was $2 \mathrm{mg} / \mathrm{l}$ and the ESR $16 \mathrm{~mm} / \mathrm{h}$ (normal $<20 \mathrm{~mm} / \mathrm{h}$ ), both within normal range. The suppression therapy was considered permanent because it had not been reasonable to replace the prosthesis.

In October 2008 the patient was well except that the knee was occasionally slightly painful. He reported that he could walk up to $1 \mathrm{~km}$ with the help of a cane. He could also climb stairs while holding onto the railing. CRP and ESR were within normal range $(2 \mathrm{mg} / \mathrm{l}$ and 8 $\mathrm{mm} / \mathrm{h}$, respectively). Oral ciprofloxacin suppression therapy was diminished to a dose of $250 \mathrm{mg}$ b.i.d. There was no limitation of extension in his prosthetic knee and the flexion was possible to $115^{\circ}$. No signs of active infection on the right knee were seen except for a slight joint swelling on palpation. The next appointment with orthopedist was scheduled for five years later, and routine follow-up was transferred to primary health care.

By the end of 2010 the patient was referred to a gastroenterologist for low hemoglobin values and occult blood in the stools. Adenocarcinoma of the transverse colon without any metastases was found and a rightsided hemicolectomy was performed with an excellent outcome. On the most recent visit in April 2011 his condition was good and no problem with the knee was evident. The ESR was $4 \mathrm{~mm} / \mathrm{h}$. The patient decided to stop the ciprofloxacin suppression therapy in the summer 2011. In February 2013 he told in a telephone consultation that the knee is still occasionally painful but not swollen, and that he could move about with a walker. An appointment with the orthopedist was scheduled for the late autumn of 2013.

\section{Discussion}

Prosthetic joint infection after total knee arthroplasty is a relatively rare complication, affecting up to $1.55 \%$ of the patients during the first two postoperative years [5]. The incidence then decreases and is $0.46 \%$ between years 2 and 10 [5]. The most common bacteria causing prosthetic joint infections are gram-positive cocci: coagulase-negative staphylococci, Staphylococcus aureus, Streptococcus spp. and Enterococcus spp. comprising about two-thirds of prosthetic knee and hip infections [6]. Aerobic gramnegative bacilli (Enterobacteriacae and Pseudomonas aeruginosa) account for up to $6 \%$ of the cases. Yersinia enterocolitica is an exceptional causative agent of prosthetic joint infection with only three previously reported cases in the English literature: one in hip [7] and two in knee joints [3,4]. We report a case of a 90-year-old man with a purulent prosthetic knee joint infection caused by Y. enterocolitica.

In 1991, Oni and Kangesu [4] reported a case of an 84year-old woman who had undergone an uncomplicated knee replacement seven years earlier. She presented with 
a six-week history of pain in her prosthetic knee on walking. The joint had become progressively swollen preventing weight-bearing for six days prior to admission. She had had no abdominal or other recent medical complaints but had become acutely febrile on the day of admission. $Y$. enterocolitica was isolated from several specimens including joint fluid and the patient was treated with total removal of the infected prosthesis and cement. Oral cefuroxime axetil $500 \mathrm{mg}$ twice daily was administered, and later reduced to $250 \mathrm{mg}$ twice daily. In 2002, Iglesias and colleagues reported a case of an 80year-old woman with a 10-year history of total knee arthroplasty [3]. Prior to admission, she had suffered from diarrhea that had resolved spontaneously. Acute symptoms included fever $\left(39^{\circ} \mathrm{C}\right)$, pain and inflammation of the affected knee. $Y$. enterocolitica was isolated from the joint fluid culture. The infection was cured by treatment with oral ciprofloxacin $750 \mathrm{mg}$ twice a day for six weeks.

There is only one report of a prosthetic hip joint infection caused by $Y$. enterocolitica [7]. The patient was a 72year-old man who had undergone hip replacement for arthrosis 10 years earlier. He had had pain in the affected hip for 5 months. Radiographs had showed loosening of the femoral component of the prosthesis. Four months later he became acutely ill with a painful and swollen thigh. All parts of the hip prosthesis including the cement and wires were replaced and the patient was administered iv cloxacillin. The swabs taken during the operation were positive for $Y$. enterocolitica and the treatment was changed to cefotaxime $1 \mathrm{~g}$ t.i.d. for 8 days. A second operation was needed because $Y$. enterocolitica could still be cultured from the drainage sites. Postoperative piperacillin $3 \mathrm{~g}$ t.i.d. was started for the first 24 hours and gentamicin $80 \mathrm{mg}$ b.i.d. for 6 days. When gentamicin was discontinued, the patient was given mecillinam 400 mg (im) t.i.d. for 14 days followed by 500 mg ciprofloxacin orally b.i.d. for another 14 days.

All three $Y$. enterocolitica prosthetic joint infections reported earlier $[3,4,7]$ have occurred several years after the initial joint replacement for arthrosis. Our patient also had a prosthetic knee joint for many years. However, the re-arthroplasty was performed seven months before the clinical manifestation of $Y$. enterocolitica infection in the prosthetic joint. All the patients, including ours were acutely ill with a high fever and an inflamed prosthetic joint. In our case, hematogenous spread of bacteria from the gut to the prosthetic knee joint is the most probable mechanism of pathogenesis. The patient reported no preceding gastrointestinal symptoms but one can assume that he had acquired the bacteria from food. Pigs are an important reservoir of $Y$. enterocolitica and eating pork without proper cooking may be a risk factor for $Y$. enterocolitica infection [2].
We cannot totally exclude the possibility of a late-onset infection originating at the time of re-arthroplasty half a year earlier. Since $Y$. enterocolitica does not typically cause perioperative contamination, this is an unlikely possibility. On the basis of previously reported cases and the present one, it is reasonable to believe that $Y$. enterocolitica can cause a late-onset prosthetic infection with acute symptoms of systemic infection through hematogenous spread.

In addition to the three previously reported prosthetic joint infections [3,4,7], there are only a few reports of deep purulent infections caused by $Y$. enterocolitica: e.g. vascular graft infections [8,9], prosthetic and native valve endocarditis [10,11], mycotic aneurysm of a carotid artery [12], lung abscess and osteomyelitis [13], meningitis [14], spondylodiscitis with or without spinal abscess [15-18]. According to the review by Crowe et al. [18] Y. enterocolitica can cause septic arthritis and osteomyelitis, as well as native valve endocarditis [11], more often than other deep sited infection.

Giamarellou et al. [11] reported a case of a 45 y-old man with a history of previous rheumatic heart disease and mitral valve prosthesis. Aortic valve insufficiency had been corrected with valvuloplasty. He presented with a spiking fever and $Y$. enterocolitica grew from his blood samples. Three months earlier, the patient had been operated for febrile appendicitis. He was initially suspected of having cholangitis because he had multiple gallbladder stones and jaundice. Later, a transesophageal echocardiogram showed the presence of a large vegetation on the mitral ring and revealed the diagnosis of prosthetic mitral valve endocarditis. Ceftriaxone $2 \mathrm{~g}$ daily in combination with gentamicin ( $80 \mathrm{mg} \mathrm{im}$, b.i.d.) was instituted and the infected mitral prosthetic valve was removed on day 27 of the treatment regimen. After the operation, antibiotic treatment was continued for two weeks. He recovered well and underwent a previously scheduled cholecystectomy.

Abad et al. [9] presented a case of a patient with an early prosthetic vascular graft infection with $Y$. enterocolitica, after repair of an abdominal aortic aneurysm. The patient was a 70-year-old man who had worked in Indonesia for 15 years on a reptile farm. An abdominal aortic aneurysm was found at clinical examination and corrected with a vascular prosthesis. Seven days after surgery the patient presented with progressive abdominal pain and fever. An ultrasound examination of the abdomen showed ileus and the presence of intra-abdominal fluid, progressing to an acute abdomen and peritonitis. A secondary laparotomy revealed pus and signs of infection of the vascular graft. Cultures of the retroperitoneal pus and the excised prosthesis grew $Y$. enterocolitica. After the re-operation, cefotaxime and ciprofloxacine were ad- 
ministered and continued for four weeks with full recovery.

Another vascular prosthesis infection with rupture was reported by Verhaegen et al. [8]. A 79-year-old man was admitted to hospital because of chills and fever. Two years earlier, a successful aortic bifurcation prosthesis operation had been performed for bilateral progressive claudication. An ultrasound examination revealed a mass surrounding the distal end of the abdominal aorta and blood cultures were positive for $Y$. enterocolitica. The patient was administered ampicillin along with gentamicin. After 16 days of intravenous therapy, the patient was discharged with oral amoxicillin. Ten days later, he was readmitted for severe low back pain and fever. Ultrasonography revealed a periaortic cystic lesion. Laparotomy was planned, but the patient died before the operation could be performed. Postmortem examination revealed a rupture of the proximal suture line of the bifurcation prosthesis.

The response to antibiotic treatment with our case was quite slow. Several weeks after the surgical operation and the commencement of antimicrobial therapy, joint swelling could still be palpated and bloody joint fluid could be repeatedly aspirated. With previous case reports on yersinia joint infections, the combination of co-trimoxazole and ciprofloxacin [3] was used with success for prosthetic knee infections, intravenous cloxacillin [7] for prosthetic hip infection, and ciprofloxacin [18] for septic arthritis caused by $Y$. enterocolitica.

Removing the infected prosthesis has been important according to the above described cases to obtain the full recovery. The ability of the bacteria to form a biofilm on the surface of the prosthesis may lead to clinical therapeutic failure in the eradication of the pathogen. Persistence of the bacterial growth in the biofilm then leads to a chronic form of infection. In the present case, not all parts of prosthesis could be removed. Taking the patient's advanced age and the severity of total re-arthroplasty into consideration, the surgeon considered debridement along with exchanging the liner components of the prosthesis as the optimal procedure for this patient. For this reason we recommended permanent suppressive antimicrobial therapy for the patient. Life-long antimicrobial therapy is a viable option for a patient over 90years. He eventually decided to stop the medication, and has fortunately had no relapses during the following two years.

Our patient as well as many others with invasive yersinia infections $[4,7,10,11,15,18]$ did not have any immunosuppressive condition unless his advanced age is considered as such. According to previous reports, some patients with invasive yersinia infection have been diabetic $[3,12]$ or alcoholic $[13,14]$. On the basis of the previous cases and the present one, it is important to re- member the aggressive nature of Yersinia enterocolitica when it manages to escape its typical environment, the gastrointestinal tract, and cause an invasive infection.

\section{Acknowledgements}

The preparation of this manuscript was supported by the Turku University Hospital and the Anne and Rauno Puolimatka Foundation. We thank Dr. Peter Dean for revising the language of the manuscript.

\section{REFERENCES}

[1] J. M. Townes, "Reactive Arthritis after Enteric Infections in the United States: The Problem of Definition," Clinical Infectious Diseases, Vol. 50, No. 2, 2010, pp. 247-254. doi:10.1086/649540

[2] E. Huovinen, L. M. Sihvonen, M. J. Virtanen, K. Haukka, A. Siitonen and M. Kuusi, "Symptoms and Sources of Yersinia enterocolitica-Infection: A Case-Control Study,” BMC Infectious Diseases, Vol. 10, 2010, pp. 122-130. doi:10.1186/1471-2334-10-122

[3] L. Iglesias, J. M. Garcia-Arenzana, A. Valiente, M. Gomariz and E. Pérez-Trallero, "Yersinia enterocolitica O:3 Infection of a Prosthetic Knee Joint Related to Recurrent Hemarthrosis," Scandinavian Journal of Infectious Diseases, Vol. 34, No. 2, 2002, pp. 132-133. doi:10.1080/00365540110080278

[4] J. A. Oni and T. Kangesu, "Yersinia enterocolitica Infection of a Prosthetic Knee Joint," British Journal of Clinical Practice, Vol. 45, No. 3, 1991, p. 225.

[5] S. M. Kurtz, K. L. Ong, E. Lau, K. J. Bozic, D. Berry and J. Parvizi, "Prosthetic Joint Infection Risk after TKA in the Medicare Population," Clinical Orthopaedics and Related Research, Vol. 468, No. 1, 2010, pp. 52-56. doi:10.1007/s11999-009-1013-5

[6] J. L. Del Pozo and R. Patel, "Infection Associated with Prosthetic Joints,” New England Journal of Medicine, Vol. 361, No. 8, 2009, pp. 787-794. doi:10.1056/NEJMcp0905029

[7] K. Hougaard and P. Søgaard, "Yersinia enterocolitica Coxitis after Hip Replacement,” Acta Orthopaedica Scandinavica, Vol. 61, No. 4, 1990, pp. 364-366. doi:10.3109/17453679008993538

[8] J. Verhagen, G. Dedeyne, W. Vansteenbergen and J. Vandepitte, "Rupture of Vascular Prothesis in a Patient with Yersinia enterocolitica Bacteremia,” Diagnostic Microbiology and Infectious Disease, Vol. 3, No. 5, 1985, pp. 451-454. doi:10.1016/0732-8893(85)90084-7

[9] C. Abad, G. Ponge and R. Elcuaz, "Early Vascular Graft Infection Due to Yersinia enterocolitica after Repair of an Abdominal Aortic Aneurysm,” Journal of Cardiovascular Surgery, Vol. 5, No. 2, 2004, pp. 161-162.

[10] E. Pras, N. Arber, J. Pinkhas and Y. Sidi, "Yersinia enterocolitica Endocarditis on a Prosthetic Valve," Postgraduate Medical Journal, Vol. 68, No. 803, 1992, pp. 762-763. doi:10.1136/pgmj.68.803.762 
[11] H. Giamarellou, A. Antoniadou, K. Kanavos, C. Papaioannou, S. Kanatakis and K. Papadaki, "Yersinia enterocolitica Endocarditis: Case Report and Literature Review," European Journal of Clinical Microbiology and Infectious Diseases, Vol. 14, 1995, No. 2, pp. 126-130.

[12] G. R. Plotkin and J. N. O’Rourke Jr., "Mycotic Aneurysm Due to Yersinia enterocolitica," American Journal of Medical Sciences, Vol. 281, No. 1, 1981, pp. 35-42. doi:10.1097/00000441-198101000-00006

[13] J. I. Sebes, E. H. Mabry Jr. and J. G. Rabinowitz, "Lung Abscess and Osteomyelitis of Rib Due to Yersinia enterocolitica," Chest, Vol. 69, No. 4, 1976, pp. 546-548. doi:10.1378/chest.69.4.546

[14] M. F. Casey, P. H. Gillian and M. L. Smiley, "Yersinia enterocolitica Meningitis and Osteomyelitis: A Case Report," Diagnostic Microbiology and Infectious Disease, Vol. 8, No. 1, 1987, pp. 47-50. doi:10.1016/0732-8893(87)90046-0

[15] M. Ellenrieder, A. E. Zautner, A. Podbielski, R. Bader and W. Mittelmeier, "Spondylodiscitis of the Lumbar Spine in a Non-Immunocompromised Host Caused by Yersinia enterocolitica O:9," Archives of Orthopaedic and Trauma Surgery, Vol. 130, No. 4, 2010, pp. 469-471. doi:10.1007/s00402-009-0921-X

[16] K. von Eckardstein, A. Spuler, C. Brauer, M. Mehl and J. Kiwit, "Spontaneous Cervical Osteomyelitis Due to Yersinia enterocolitica in a Non-Immunocompromised Host," European Journal of Clinical Microbiology and Infectious Diseases, Vol. 23, No. 1, 2004, pp. 66-68. doi:10.1007/s10096-003-1065-5

[17] J. T. Sinnott 4th, H. Multhopp, J. Leo and G. Rechtine, "Yersinia enterocolitica Causing Spinal Osteomyelitis and Empyema in a Nonimmunocompromised Host," Southern Medical Journal, Vol. 82, No. 3, 1989, pp. 399-400. doi:10.1097/00007611-198903000-00033

[18] M. Crowe, K. Ashford and P. Ispahani, “Clinical Features and Antibiotic Treatment of Septic Arthritis and Osteomyelitis Due to Yersinia enterocolitica," Journal of Medical Microbiology, Vol. 45, No. 4, 1996, pp. 302-309. doi:10.1099/00222615-45-4-302 